\title{
Fredholm İntegro Diferansiyel Denklemin Sayısal Çözümü için Alternatif Bir Yöntem
}

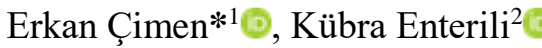 \\ ${ }^{1}$ Van Yüzüncü Y1l Üniversitesi, Eğitim Fakültesi, Matematik Bölümü, Van, TÜRKİYE \\ ${ }^{2}$ Van Yüzüncü Yıl Üniversitesi, Fen Bilimleri Enstitüsü, Van, TÜRKIYYE
}

Geliş / Received: 16/10/2019, Kabul / Accepted: 20/02/2020

\begin{abstract}
Öz
$\mathrm{Bu}$ çalışmada, birinci mertebeden lineer Fredholm integro diferansiyel denklem için başlangıç değer problemini ele alıyoruz. Bu problemin nümerik çözümü için düzgün şebekede bir yeni fark şeması inşa ediyoruz. Bu şema, kalan terimi integral biçiminde olan interpolasyon quadratür formülleri ve üstel baz fonksiyonunu içeren integral özdeşliklerinden meydana gelmektedir. Metodun ayrık maksimum normda birinci mertebeden yakınsaklığı ispatladık. Ayrıca, hem sunulan metot hem de Euler metodu kullanılarak bir örnek çözüldü ve hesaplanan sonuçlar kaşılaştırıldı.
\end{abstract}

Anahtar Kelimeler: Fredholm integro-diferansiyel denklem, başlangıç-değer problemi, sonlu fark metodu, hata değerlendirmesi.

\section{An Alternative Method for Numerical Solution of Fredholm Integro Differential Equation}

\begin{abstract}
In this paper, we consider a linear first order Fredholm integro differential equation with initial condition. To solve this problem numerically, we construct a new difference scheme on a uniform mesh. The scheme is based on the method of integral identities with the use of exponential basis functions and interpolating quadrature rules with the weight and remainder terms in integral form. We prove that the method is first order convergence in the discrete maximum norm. Moreover, a numerical example is solved using both the presented method and the Euler method and compared the computed results.
\end{abstract}

Keywords: Fredholm integro-differential equation, initial value problem, finite difference method, error estimate

\section{Giriş}

İntegral denklemler fen ve mühendislik bilimlerinin yanı sıra teknik alandaki uygulamalarda yaygin olarak ortaya çıkmaktadır. Özellikle elastik teori, biomekanik, elektromagnetik, akışkanlar dinamiği, 1S1 transferi, popülasyon dinamiği, enfeksiyon hastalıklarının yayılması gibi fizik ve biyoloji alanlarındaki modellere sıkça rastlanmaktadır (Bloom, 1980; Holmaker, 1993; Forbes vd., 1997; Jerri,

*Sorumlu Yazar: cimenerkan@hotmail.com 
1999; Medlock ve Kot, 2003; Rahman, 2007; Wazwaz, 2011).

İntegral ve integro diferansiyel denklemlerin çözümü için yapılan çalışmalar Volterra'dan günümüze halen devam etmektedir (Volterra, 1959). $\mathrm{Bu}$ denklemlerle ilgili çalışmalar lineer denklemleri içerse de, çoğu zaman bunların analitik çözümlerini bulmak mümkün olmamaktadır. Bu nedenle nümerik yaklaşımlar literatürde daha fazla yer bulmaktadır. Örneğin, dikdörtgen metodu, yamuk metodu, Simpson metodu gibi geleneksel metotlar sıklıkla kullanılmaktadır (Hackbusch, 1995; Jerri, 1999; Kythe ve Puri, 2002). Bununla birlikte, son y1llarda birçok yazarın; bölge ayrıştırma metodu, Taylor seri metodu, dalgaciklar (wavelets) yöntemi, doğuran çekirdekli Hilbert metodu ve sonlu farklar metodu gibi yeni metotları kullandıkları görülmektedir (Darania ve Ebadian, 2007; Lakestani vd., 2011; Maleknejad ve Attary, 2011; Wazwaz, 2011; Arqub vd., 2013; Pandey, 2015; Amiraliyev vd., 2018; Çimen, 2018; Yapman vd., 2019).

$\mathrm{Bu}$ çalışmada, aşağıdaki Fredholm integro diferansiyel denklemi için başlangıç değer problemini ele alacağız:

$$
\begin{aligned}
L u \equiv & u^{\prime}(x)+a(x) u(x)=f(x) \\
& +\lambda \int_{0}^{l} K(x, t) u(t) d t, x \in \Omega, \\
& u(0)=\mu,
\end{aligned}
$$

burada $\Omega=(0, l]$ ve $\mu$ verilmiş reel bir sabittir. $\quad a(x) \geq \alpha>0, \quad f(x)$ ve $K(x, t)$ sirasiyla, $\bar{\Omega}$ ve $\bar{\Omega} \times \bar{\Omega}$ üzerinde istenilen türevlere sahip sürekli fonksiyonlardır. Çalışmanın 2. Bölümü'nde problemin analitik çözümünün bazı özelliklerini inceleyeceğiz. Sonraki bölümde sonlu fark metodunu kullanarak uygun fark şemasını elde edeceğiz. Daha sonra fark probleminin yakınsaklık ve kararlılık gibi temel özelliklerini inceleyeceğiz. Son bölümde elde ettiğimiz teorik sonuçları doğrulayan bir örnek vereceğiz.

Dahası, çalışmamız boyunca $C$ genel pozitif sabit ve $\|g\|_{\infty}$ ise herhangi sürekli bir $g$ fonksiyonu için sürekli maksimum norm olarak alınmaktadır.

\section{Analitik Çözümün Bazı Özellikleri}

$\mathrm{Bu}$ bölümde, uygun nümerik çözümün analizi için ihtiyaç duyacağımız ve sonraki bölümlerde kullanacağımız (1.1)-(1.2) probleminin analitik çözümünün bazı önemli özelliklerini vereceğiz.

Lemma 2.1: $a(x), f(x) \in C(\bar{\Omega}), K(x, t) \in$ $C(\bar{\Omega} \times \bar{\Omega}) \mathrm{ve}$

$$
\delta=\alpha^{-1}|\lambda| \max _{x \in \bar{\Omega}} \int_{0}^{l}|K(x, t)| d t<1,
$$

olsun. $\mathrm{Bu}$ durumda (1.1)-(1.2) probleminin analitik çözümü olan $u$ için aşağıdaki değerlendirmeler doğrudur:

$$
\begin{aligned}
& \|u\|_{\infty} \leq C_{0}, \\
& \left\|u^{\prime}\right\|_{\infty} \leq\left(|\lambda| \bar{K} l+\|a\|_{\infty}\right) C_{0}+\|f\|_{\infty},
\end{aligned}
$$

burada

$$
\begin{gathered}
C_{0}=\left(|\mu|+\alpha^{-1}\|f\|_{\infty}\right)(1-\delta)^{-1}, \\
\bar{K}=\max _{(x, t) \in \bar{\Omega} \times \bar{\Omega}}|K(x, t)|,
\end{gathered}
$$

biçimindedir.

İspat: (1.1) denkleminden,

$$
\begin{aligned}
u(x)= & \mu e^{-\int_{0}^{x} a(t) d t}+\int_{0}^{x}[f(s) \\
& \left.+\lambda \int_{0}^{l} K(s, t) u(t) d t\right] e^{-\int_{s}^{x} a(t) d t} d s,
\end{aligned}
$$

yazabiliriz. Buradan

$$
\begin{gathered}
|u(x)| \leq|\mu| e^{-\alpha x}+\int_{0}^{x}|f(s)| e^{-\alpha(x-s)} d s \\
+|\lambda| \int_{0}^{x}\left[\int_{0}^{l}|K(s, t)||u(t)| d t\right] e^{-\alpha(x-s)} d s \\
\leq|\mu|+\alpha^{-1}\|f\|_{\infty}\left(1-e^{-\alpha x}\right)+|\lambda|\|u\|_{\infty} \\
\quad \times \max _{0 \leq s \leq l} \int_{0}^{l}|K(s, t)| d t \alpha^{-1}\left(1-e^{-\alpha x}\right),
\end{gathered}
$$

elde ederiz. Buradan (2.1)'in doğruluğu kolayca görülür. Yine (1.1)'den 


$$
\begin{aligned}
\left|u^{\prime}(x)\right| & \leq|a(x)||u(x)|+|f(x)| \\
& +|\lambda| \int_{0}^{l}|K(x, t)||u(t)| d t,
\end{aligned}
$$

yazabiliriz. Buradan,

$$
\begin{gathered}
\left|u^{\prime}(x)\right| \leq\|a\|_{\infty}\|u\|_{\infty}+\|f\|_{\infty} \\
+|\lambda| \bar{K}\|u\|_{\infty} l,
\end{gathered}
$$

elde ederiz. $\mathrm{Bu}$ ise (2.2)'nin doğruluğunu gösterir.

\section{Fark Şeması}

Bu bölümde (1.1)-(1.2) probleminin nümerik çözümü için uygun fark problemini elde edeceğiz. Bunun için öncelikle fark probleminde kullanacağımı bazı gösterimleri verelim. $\omega_{N}, \bar{\Omega}$ üzerinde bir düzgün şebeke olsun:

$$
\begin{gathered}
\omega_{N}=\left\{x_{i}=i h, i=1,2, \ldots, N ; h=l / N\right\}, \\
\bar{\omega}_{N}=\omega_{N} \cup\{0\} .
\end{gathered}
$$

Ayrıca $\omega_{N}$ üzerinde herhangi bir $g(x)$ şebeke fonksiyonu için $g_{i}=g\left(x_{i}\right)$ ve $u(x)$ 'in $x_{i}$ noktasındaki yaklaşımını da $y_{i}$ olarak alacağız. Yine, bu $g_{i}$ şebeke fonksiyonu için

$$
g_{\bar{x}, i}=\frac{g_{i}-g_{i-1}}{h},
$$

geri fark türevi ve

$$
\|g\|_{\infty, \omega_{N}}=\max _{1 \leq i \leq N}\left|g_{i}\right|,
$$

gösterimlerini kullanacağız.

Fark şemasının kurulmasında ilk olarak aşağıdaki özdeşlikten yararlanacağız:

$$
\begin{aligned}
& h^{-1} \int_{x_{i-1}}^{x_{i}} L u(x) \varphi_{i}(x) d x=h^{-1} \int_{x_{i-1}}^{x_{i}}[f(x) \\
& \left.\quad+\lambda \int_{0}^{l} K(x, t) u(t) d t\right] \varphi_{i}(x) d x, 1 \leq i \leq N,
\end{aligned}
$$

burada $\varphi_{i}(x)$ :

$$
\varphi_{i}(x)=e^{-\int_{x}^{x_{i}} a(t) d t}, x_{i-1} \leq x \leq x_{i},
$$

olarak belirlenmiş baz fonksiyonu,

$$
\left\{\begin{array}{l}
-\varphi_{i}{ }^{\prime}(x)+a(x) \varphi_{i}(x)=0, x_{i-1}<x \leq x_{i} \\
\varphi_{i}\left(x_{i}\right)=1
\end{array}\right.
$$

probleminin çözümüdür.

(3.1) bağıntısını yeniden düzenlersek,

$$
\begin{aligned}
& h^{-1} \int_{x_{i-1}}^{x_{i}} u^{\prime}(x) \varphi_{i}(x) d x \\
& \quad+h^{-1} \int_{x_{i-1}}^{x_{i}} a(x) u(x) \varphi_{i}(x) d x
\end{aligned}
$$

$$
\begin{aligned}
=h^{-1} \int_{x_{i-1}}^{x_{i}} f(x) \varphi_{i}(x) d x \\
\quad+h^{-1} \int_{x_{i-1}}^{x_{i}}\left[\lambda \int_{0}^{l} K(x, t) u(t) d t\right] \varphi_{i}(x) d x,
\end{aligned}
$$

yazabiliriz. Daha sonra Amiraliyev ve Mamedov'un (1995) çalışmasında bulunan (2.1) ve (2.2) formüllerini $\left(x_{i-1}, x_{i}\right)$ alt aralığ için (3.3) denkleminin sol tarafında dikkate alırsak,

$$
\begin{aligned}
& h^{-1} \int_{x_{i-1}}^{x_{i}} u^{\prime}(x) \varphi_{i}(x) d x \\
& \quad+h^{-1} \int_{x_{i-1}}^{x_{i}} a(x) u(x) \varphi_{i}(x) d x \\
& \quad=A_{i} u_{\bar{x}, i}+B_{i} u_{i}
\end{aligned}
$$

elde ederiz. Burada

$$
\begin{aligned}
A_{i}= & h^{-1} \int_{x_{i-1}}^{x_{i}} \varphi_{i}(x) d x \\
& +h^{-1} \int_{x_{i-1}}^{x_{i}}\left(x-x_{i}\right) a(x) \varphi_{i}(x) d x, \\
B_{i} & =h^{-1} \int_{x_{i-1}}^{x_{i}} a(x) \varphi_{i}(x) d x
\end{aligned}
$$

biçimindedir. Şimdi de (3.3) denkleminin sağ tarafındaki integral terimine uygun quadratür formüllerini uygularsak,

$h^{-1} \int_{x_{i-1}}^{x_{i}}\left[\lambda \int_{0}^{l} K(x, t) u(t) d t\right] \varphi_{i}(x) d x$

$=h^{-1} \lambda \int_{x_{i-1}}^{x_{i}} \varphi_{i}(x) d x \int_{0}^{l} K\left(x_{i}, t\right) u(t) d t+$

$R_{i}^{(1)}$

$=h^{-1} \lambda \int_{x_{i-1}}^{x_{i}} \varphi_{i}(x) d x h \sum_{j=1}^{N} K\left(x_{i}, t_{j}\right) u_{j}+$

$R_{i}^{(1)}+R_{i}^{(2)}$,

elde ederiz. Burada 
$R_{i}^{(1)}=\lambda h^{-1} \int_{x_{i-1}}^{x_{i}} \varphi_{i}(x) d x$

$\times \int_{x_{i-1}}^{x_{i}} T_{0}(\xi-x)\left(\int_{0}^{l} \frac{\partial}{\partial \xi} K(\xi, t) u(t) d t\right) d \xi$,

$R_{i}^{(2)}=\lambda h^{-1} \int_{x_{i-1}}^{x_{i}} \varphi_{i}(x) d x$

$\times \sum_{j=1}^{N} \int_{x_{j-1}}^{x_{j}}\left(s-x_{j-1}\right) \frac{d}{d s}\left[K\left(x_{i}, s\right) u(s)\right] d s$,

$$
T_{0}(t)=\left\{\begin{array}{l}
1, t>0 \\
0, t \leq 0
\end{array}\right.
$$

biçimindedir. Buradan, $u\left(x_{i}\right)$ için

$$
\begin{aligned}
\ell u_{i}:= & A_{i} u_{\bar{x}, i}+B_{i} u_{i}=F_{i} \\
& +C_{i} \lambda h \sum_{j=1}^{N} K\left(x_{i}, t_{j}\right) u_{j}+R_{i},
\end{aligned}
$$

kesin fark şemasını yazabiliriz. Burada

$$
\begin{aligned}
C_{i} & =h^{-1} \int_{x_{i-1}}^{x_{i}} \varphi_{i}(x) d x, \\
F_{i} & =h^{-1} \int_{x_{i-1}}^{x_{i}} f(x) \varphi_{i}(x) d x, \\
R_{i} & =R_{i}^{(1)}+R_{i}^{(2)},
\end{aligned}
$$

$A_{i}, B_{i}$ ve $R_{i}^{(k)}(k=1,2)$ ifadeleri sirasiyla (3.4)-(3.6) denklemlerinde belirtilmektedir.

Böylece, (3.7) bağıntısından (1.1)-(1.2) probleminin yaklaşık çözümü için aşağıdaki fark şemasını önerebiliriz:

$$
\begin{aligned}
& \ell y_{i}:=A_{i} y_{\bar{x}, i}+B_{i} y_{i}=F_{i} \\
& \quad+C_{i} \lambda h \sum_{j=1}^{N} K\left(x_{i}, t_{j}\right) y_{j}, 1 \leq i \leq N, \\
& \quad y_{0}=\mu .
\end{aligned}
$$

Bununla birlikte, Euler metodu ve dikdörtgen metodunu kullanarak aşağıdaki şemayı kolayca elde edebiliriz:

$$
\begin{array}{ll}
y_{\bar{x}, i}+a_{i} y_{i}=f_{i}+\lambda h \sum_{j=1}^{N} K\left(x_{i}, t_{j}\right) y_{j} \\
& 1 \leq i \leq N, \\
y_{0}=\mu . &
\end{array}
$$

\section{Yakınsaklık Analizi}

Metodun yakınsaklığını araştırmak için,

$$
\begin{aligned}
\ell z_{i} & =R_{i}, 1 \leq i \leq N, \\
z_{0} & =0,
\end{aligned}
$$

probleminin çözümü olan $z_{i}=y_{i}-u_{i},(1 \leq$ $i \leq N)$ hata fonksiyonunu tanımlayalım. Burada $R_{i}$ (3.5)-(3.6) denklemlerinde ifade edilen kalan terimlerin toplamıdır.

Lemma 4.1: Eğer $a(x), f(x) \in C(\bar{\Omega})$, $K(x, t) \in C^{1}(\bar{\Omega} \times \bar{\Omega})$ ise, $R_{i}$ kalan terimi için

$$
\|R\|_{\infty, \omega_{N}} \leq C h,
$$

değerlendirmesi doğrudur.

İspat: $0<\varphi_{i}(x) \leq 1$ ve $\left|\frac{\partial}{\partial \xi} K(\xi, t)\right| \leq C$ eşitsizliklerini (3.5) denkleminde dikkate alırsak,

$$
\left|R_{i}^{(1)}\right| \leq C|\lambda| \int_{x_{i-1}}^{x_{i}}\left(\int_{0}^{l} u(t) d t\right) d \xi,
$$

ve Lemma 2.1'den

$\left|R_{i}^{(1)}\right| \leq C|\lambda| \int_{x_{i-1}}^{x_{i}}\left(C_{0} l\right) d \xi=O(h)$.

(3.6)'dan ve $0<\varphi_{i}(x) \leq 1$ eşitsizliğinden,

$\left|R_{i}^{(2)}\right| \leq C|\lambda| \sum_{j=1}^{N} \int_{x_{j-1}}^{x_{j}}\left|s-x_{j-1}\right|$

$\times\left[\left|\frac{d K\left(x_{i}, s\right)}{d s}\right||u(s)|+\left|K\left(x_{i}, s\right)\right|\left|u^{\prime}(s)\right|\right] d s$,

yazabiliriz. Lemma 2.1 ve $\left|\frac{\partial}{\partial s} K(x, s)\right| \leq C$ eşitsizliğinden,

$\left|R_{i}^{(2)}\right| \leq C \sum_{j=1}^{N} \int_{x_{j-1}}^{x_{j}}\left|s-x_{j-1}\right| d s=C h^{2} N$, elde ederiz. 
Lemma 4.2: Azalmayan $\bar{G}_{i}$ fonksiyonu için $\left|G_{i}\right| \leq \bar{G}_{i}$ ve

$$
\begin{aligned}
\ell v_{i} & :=A_{i} v_{\bar{x}, i}+B_{i} v_{i}=G_{i}, 1 \leq i \leq N, \\
v_{0} & =\beta,
\end{aligned}
$$

olsun. $\mathrm{Bu}$ durumda (4.3)-(4.4.) fark problemi için

$$
\left|v_{i}\right| \leq \beta+\alpha^{-1} \bar{G}_{i}, 1 \leq i \leq N,
$$

değerlendirmesi doğrudur (Amiraliyev ve Y1lmaz, 2014).

Lemma 4.3: $z_{i}, \quad(4.1)-(4.2)$ probleminin çözümü olsun ve

$$
\gamma=\alpha^{-1}|\lambda| \max _{1 \leq i \leq N} \sum_{j=1}^{N}\left|K\left(x_{i}, t_{j}\right)\right|<1,
$$

şartı sağlansın. Bu durumda

$$
\|z\|_{\infty, \omega_{N}} \leq(1-\gamma)^{-1}\|R\|_{\infty, \omega_{N}},
$$

eşitsizliği doğrudur.

İspat: (4.1) denkleminden,

$$
\begin{aligned}
A_{i} z_{\bar{x}, i} & +B_{i} z_{i}= \\
& +C_{i} \lambda h \sum_{j=1}^{N} K\left(x_{i}, t_{j}\right) z_{j}+R_{i},
\end{aligned}
$$

yazabiliriz. Lemma 4.2'yi ve (4.2) şartını dikkate alırsak,

$$
\begin{gathered}
\|z\|_{\infty} \leq \alpha^{-1}|\lambda|\|z\|_{\infty} \max _{1 \leq i \leq N} \sum_{j=1}^{N}\left|K\left(x_{i}, t_{j}\right)\right| \\
+\|R\|_{\infty},
\end{gathered}
$$

elde ederiz.

Son olarak, yukarıdaki lemmaları bir arada düşünerek, metodun yakınsaklık sonucunu ifade eden teoremi verelim.

Teorem 4.1: $u, \quad(1.1)-(1.2)$ probleminin analitik çözümü ve $y$, (3.8)-(3.9) fark probleminin çözümü olsun. Bu durumda değerlendirmesi doğrudur.

\section{Nümerik Sonuçlar}

Şimdi de aşağıdaki problemi ele alalım:

$$
\begin{aligned}
u^{\prime}(x) & +2 u(x)=\frac{1}{4}\left(e^{-(1+x)}-e^{-x}\right) \\
& +\frac{1}{4} \int_{0}^{1} e^{t-x} u(t) d t, x \in(0,1],
\end{aligned}
$$

$u(0)=1$.

Bu problemin analitik çözümü $u(x)=e^{-2 x}$.

\begin{tabular}{|c|c|c|c|}
\hline$x_{i}$ & $u_{i}$ & $y_{i}$ & $\left|y_{i}-u_{i}\right|$ \\
\hline 0.125 & 0.7788008 & 0.7785212 & $2.796 \mathrm{E}-4$ \\
\hline 0.250 & 0.6065307 & 0.6060662 & $4.645 \mathrm{E}-4$ \\
\hline 0.375 & 0.4723666 & 0.4717871 & $5.795 \mathrm{E}-4$ \\
\hline 0.500 & 0.3678794 & 0.3672360 & $6.435 \mathrm{E}-4$ \\
\hline 0.625 & 0.2865048 & 0.2858341 & 6.707 E-4 \\
\hline 0.750 & 0.2231302 & 0.2224582 & $6.720 \mathrm{E}-4$ \\
\hline 0.875 & 0.1737739 & 0.1731185 & $6.554 \mathrm{E}-4$ \\
\hline 1.000 & 0.1353353 & 0.1347083 & 6.270 E-4 \\
\hline
\end{tabular}

Bu problem için (3.8)-(3.9) fark problemi $y_{i}$, $(1 \leq i \leq N)$ bilinmeyenlerine göre bir lineer denklem sistemi olarak düşünülebilir. $\mathrm{Bu}$ sistem, N'nin farklı değerleri için Mathematica programı yardımıyla çözülmüştür. Gerek sunulan metot (SM), gerekse klasik Euler metodu (EM) ile elde edilen sonuçlar aşağıdaki tablolarda listelenmiş ve bir grafikte verilmiştir.

\begin{tabular}{llll}
\multicolumn{4}{c}{ Tablo 1. Nümerik sonuçlar $(N=64)(\mathrm{SM})$} \\
\hline$x_{i}$ & $u_{i}$ & $y_{i}$ & $\left|y_{i}-u_{i}\right|$ \\
\hline 0.125 & 0.7788008 & 0.7785212 & $2.796 \mathrm{E}-4$ \\
0.250 & 0.6065307 & 0.6060662 & $4.645 \mathrm{E}-4$ \\
0.375 & 0.4723666 & 0.4717871 & $5.795 \mathrm{E}-4$ \\
0.500 & 0.3678794 & 0.3672360 & $6.435 \mathrm{E}-4$ \\
0.625 & 0.2865048 & 0.2858341 & $6.707 \mathrm{E}-4$ \\
0.750 & 0.2231302 & 0.2224582 & $6.720 \mathrm{E}-4$ \\
0.875 & 0.1737739 & 0.1731185 & $6.554 \mathrm{E}-4$ \\
1.000 & 0.1353353 & 0.1347083 & $6.270 \mathrm{E}-4$ \\
\hline
\end{tabular}

$$
\|y-u\|_{\infty, \omega_{N}} \leq C h,
$$


Tablo 3. Her iki metot için maksimum hata değerlendirmesi

\begin{tabular}{lll}
\hline$N$ & $e^{N}(\mathrm{EM})$ & $e^{N}(\mathrm{SM})$ \\
\hline 32 & $1.157 \mathrm{E}-2$ & $1.337 \mathrm{E}-3$ \\
64 & $5.875 \mathrm{E}-3$ & $6.740 \mathrm{E}-4$ \\
128 & $2.960 \mathrm{E}-3$ & $3.385 \mathrm{E}-4$ \\
256 & $1.486 \mathrm{E}-3$ & $1.696 \mathrm{E}-4$ \\
512 & $7.444 \mathrm{E}-4$ & $8.489 \mathrm{E}-5$ \\
1024 & $3.725 \mathrm{E}-4$ & $4.247 \mathrm{E}-5$ \\
\hline
\end{tabular}

$e^{N}=\max _{1 \leq i \leq N}\left|y_{i}-u_{i}\right|$

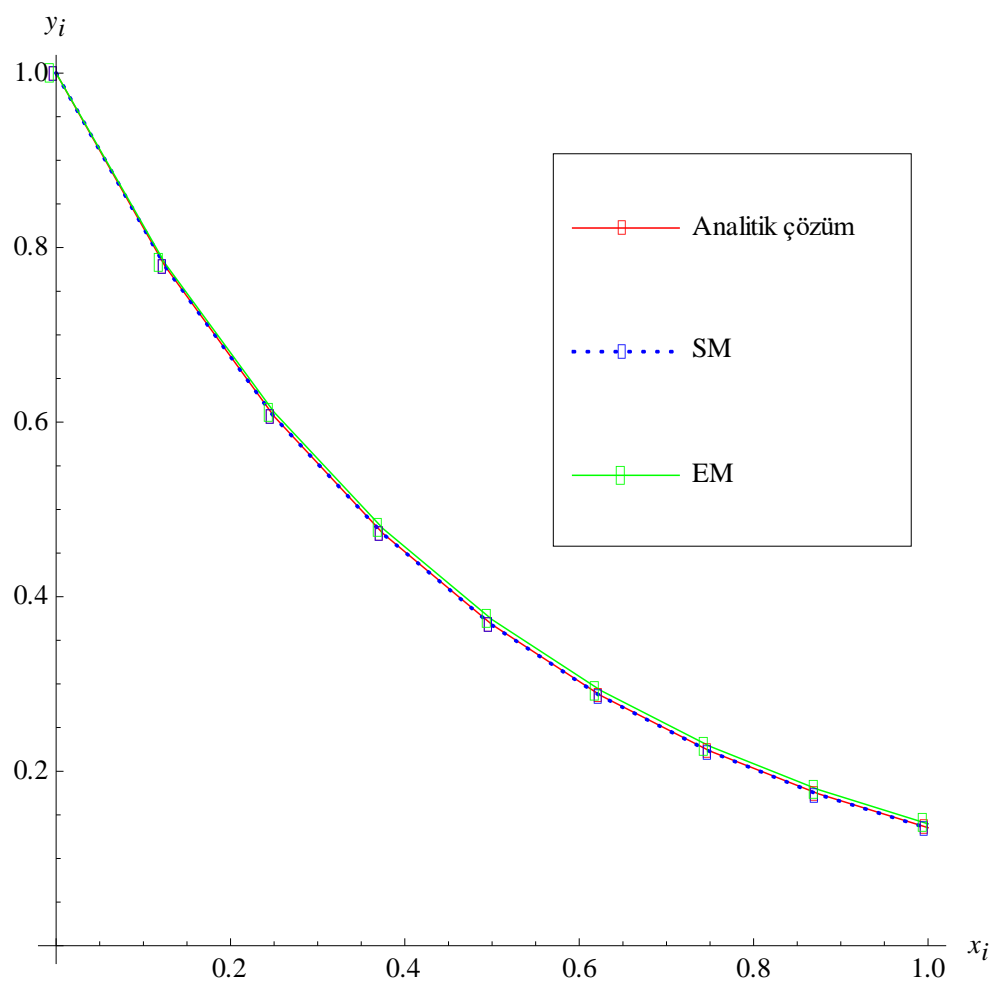

Şekil 1. (5.1)-(5.2) probleminin $N=64$ için analitik ve nümerik sonuçları.

\section{Sonuç}

$\mathrm{Bu}$ çalışmada birinci mertebeden lineer Fedholm integro-diferansiyel denklem için başlangıç değer problemi ele alınmıştır. Bu problemin nümerik çözümü için katsayıları integral biçiminde olan ve kalan terimi integral terimi içeren quadratür formülleri yardımıyla yeni bir fark şeması elde edilmiştir. (1.1)-(1.2) problemine uygun bir örnek verilerek, yeni şema ve klasik şema kullanılarak elde edilen sonuçlar Tablo 1.2.'de sunulmuştur. Tablo 3.'de ise her iki şemanın $N=32,64, \ldots, 1024$ için maksimum hataları karşılaştırılmıştır. Ayrıca Şekil 1. incelendiğinde yeni yöntemin klasik Euler yöntemine kıyasla daha iyi sonuçlar verdiği görülmüştür. $\mathrm{Bu}$ çalışmadan elde edilen sonuçlar, gecikmeli integro-diferansiyel denklemlerin nümerik çözümü için yol göstericidir. 


\section{Kaynaklar}

Amiraliyev G. M., Durmaz M. E., Kudu M. 2018. "Uniform convergence results for singularly perturbed Fredholm integrodifferential equation", J. Math. Anal. 9(6), 55-64.

Amiraliyev G. M., Mamedov Y. D. 1995. "Difference schemes on the uniform mesh for a singularly perturbed pseudo-parabolic equations", Tr. J. Math. 22, 202-222.

Amiraliyev, G.M., Yilmaz, B. 2014. "Fitted difference method for a singularly perturbed initial value problem", Int. J. Math. Comput., 22(1), 1-10.

Arqub O. A., Al-Smadi M., Shawagfeh N. 2013. "Solving Fredholm integro-differential equations using reproducing kernel Hilbert space method", Appl. Math. Comput. 219, 8938-8948.

Bloom F. 1980. "Asymptotic bounds for solutions to a system of damped integrodifferential equations of electromagnetic theory", J. Math. Anal. Appl. 73, 524-542.

Çimen E. 2018. "A computational method for Volterra integro-differential equation", Erzincan Üniversitesi Fen Bilimleri Enstitüsü Dergisi, 11(3), 347-352.

Darania P., Ebadian A. 2007. “A method for the numerical solution of the integrodifferential equations", Appl. Math. Comput. 188(1), 657-668.

Forbes L. K., Crozier S., Doddrell D. M. 1997. "Calculating current densities and fields produced by shielded magnetic resonance imaging probes", SIAM J. Appl. Math. 57, 401-425.
Hackbusch W. (1995). "Integral Equations Theory and Numerical Treatment", Birkhauser, Basel.

Holmaker K. 1993. "Global asymptotic stability for a stationary solution of a system of integro-differential equations describing the formation of liver zones", SIAM J. Math. Anal. 24, 116-128.

Jerri A. (1999). "Introduction to Integral Equations with Applications", Wiley, New York.

Kythe P. K., Puri P. (2002). "Computational Methods for Linear Integral Equations", Springer, New York.

Lakestani M., Saray B. M., Dehghan, M. (2011). "Numerical solution for the weakly singular Fredholm integro-differential equations using Legendre multiwavelets", $J$. Comput. Appl. Math. 235, 3291-3303.

Maleknejad K., Attary, M. (2011). "An efficient numerical approximation for the linear class of Fredholm integro-differential equations based on Cattani's method", Commun. Nonlinear Sci. Numer. Simulat. 16, 2672-2679.

Medlock J., Kot M. 2003. "Spreading disease: integro-differential equations old and new", Math. Biosciences. 184, 201-222.

Pandey P. K. (2015). "Numerical solution of linear Fredholm integro-differential equations by non-standard finite difference method ", Appl. Appl. Math. 10(2), 10191026.

Rahman M. (2007). "Integral Equations and Their Applications", WIT Press, Boston. 
Volterra V. (1959). “Theory of Functionals and of Integral and Integro-differential Equations", Dover Publications, New York.

Wazwaz A. M. (2011). "Linear and Nonlinear Integral Equations Methods and Applications", Springer, Berlin.

Yapman Ö., Amiraliyev G. M., Amirali I. 2019. "Convergence analysis of fitted numerical method for a singularly perturbed nonlinear Volterra integro-differential equation with delay". J. Comput. Appl. Math. 355, 301-309. 